\title{
Digital twins are shaping future virtual worlds
}

\author{
Jingzhi Guo ${ }^{1}$
}

Accepted: 11 March 2021 / Published online: 26 March 2021

(c) The Author(s), under exclusive licence to Springer-Verlag London Ltd., part of Springer Nature 2021

\section{Introduction}

With the in-depth research of digital twins in industries, especially in manufacturing, digital twins have been gradually applied in more fields such as e-commerce, e-learning, healthcare, virtual office, and remote control of unmanned exploration [1-5]. These phenomena show that the concept of digital twin is rapidly penetrating into the traditional aspects of planning, simulation, prediction, process design, and service fusion. Researchandmarkets.com reported that the global digital twin market share would have a $31.9 \%$ annual increase between 2020 and 2030, and the revenue generated would rise from US\$3.6 billion in 2019 to US $\$ 73$ billion by 2030. Meanwhile, grandviewresearch. com reported that the market size value of virtual reality, a key supporting tool of virtual world, was US\$15.8 billion in 2020 and would reach to US\$62.1 billion in forecast at a rate of $21.6 \%$ from 2020 to 2027 . These indicate a driving force pushing digital twins toward virtual worlds.

\section{Challenges of digital twins}

A digital twin often refers to a digital representation of a physical twin that is a real-world entity, system, or thing [1, 6]. It implements itself as an encapsulated software object or model that mirrors a unique object, process, organization, person, and legendary story, no matter whether such a thing is concrete or abstract in real world. When multiple digital twins integrate together, they become a composite digital twin.

Things in the real world are often complex with the characteristics of clustering and collaboration. Spatially, things group themselves into many different combinations.

Jingzhi Guo

jzguo@um.edu.mo

1 Faculty of Science and Technology, University of Macau, Macau, China
Temporally, things trigger events, produce states, while they reach certain conditions. There are interactions of humanto-human, human-to-thing, and thing-to-thing, which create many complex relations and processes. As the mirrors of real-world things, digital twins also share the same characteristics and require the modeling of digital objects and processes with capabilities of composition, collaboration, flexibility, sensing ability, and simulation. To have these capabilities, digital twins have to meet some challenges as follows.

- A digital twin should be able to exactly capture its physical properties, accurately simulate its behaviors, and adjust its size to model both simple objects (e.g., car, plane, and a person), and complex objects and relations (e.g., an irrigation system, shopping process, store, city, and earth [7]).

- Digital twins should be able to work collaboratively with each other. For example, inside of a composite digital twin, digital twins can well work together. Externally, a digital twin can cooperatively work and interact with other digital twins in different contexts.

- A digital twin should be self-conscious about its changes of size and functionality in real time, and modify itself when needed.

- A digital twin should be able to sense the ambient events in time and respond with state changes on conditions. It should also be able to detect the execution conflicts and resolve them for consistent communications.

- A digital twin can embody a human user as an avatar to interact with other avatars or digital twins immersively.

\section{Supporting technologies of digital twins toward future virtual worlds}

To meet the challenges of digital twins, recent researches are emphasizing on model flexibility, integration \& fusion, real-time responsiveness, environment sensing, consistent semantics, and immersive interaction. These changes 
have created many composite digital twins that are closely interacting with each other in two levels, in which a future virtual world is emerging as a cyber-physically integrated world. Specifically, the two levels of the world are as follows:

First, a digital twin level is constructed by many connected digital twins. It consists of object digital twins (i.e., digital objects such as sky, tree, building, equipment, product, and ad board) and process digital twins (i.e., digital processes such as shopping, drone patrol, product lifecycle, simulation, and experiment) in the sense of interactive relations between digital objects, which are exactly there in real world. The digital twins of this level have unique mappings onto their corresponding physical twins that could be realworld things and relations between things.

Second, a virtual twin level is constructed by many connected virtual twins. It consists of non-real-world object virtual twins (i.e., virtual objects such as space warship created from fantasies and chatting room digitally created), person virtual twins (i.e., virtual avatars constructed from human users or softbot users), and process virtual twins (i.e., virtual processes such as virtual shopping, virtual class-attending, and virtual chatting that might behave differently as in real world). This level depicts the virtual behaviors of object-toobject, object-to-avatar, and avatar-to-avatar. There are no mapping relationships between virtual twins and real-world things. Contrarily, there is a mapping relationship between virtual twins and the things imagined in the mind worlds of human users. These imaginations can be realized as the virtual twins with no relationship to digital twins. They can also be implemented as the virtual twins interactable with digital twins to form virtual applications, where digital twins and virtual twins work together in a same interactive space. The fusion of physical twins, digital twins, and virtual twins signifies a big move toward a future virtual world, where the core supporting technologies are as follows:

\section{- Technologies for digital twin level}

Sensor technology collects data from real-world devices and systems through digital twins and controls feedbacks.

Digital model construction is the digital modeling by mapping the shape and laws of a real-world thing through geometric modeling, white-box modeling, and data modeling. Digital model interoperability is the fusion of geometric, white-box, and data models for digital twins.

\section{- Technologies for virtual twin level}

Virtual model construction \& interoperability: virtual modeling and interoperability as digital modeling.

\section{- Technologies for both digital \& virtual twin levels}

Human-computer interaction enables human users to interact with digital and virtual objects, and virtual avatars through computing devices.

Process management plans, designs, and manages digital processes and virtual processes that satisfy goals.

Data integration seamlessly connects heterogeneous devices in real world, heterogeneous digital objects in digital twin level, and heterogeneous virtual objects in virtual twin level. Process integration seamlessly connects heterogeneous processes in real world, heterogeneous digital processes in digital twin level, and heterogeneous virtual processes in virtual twin level.

\section{Technological trends of digital twins toward future virtual worlds}

While more and more industries introduce digital twins into their areas, new technologies, such as Industrial Internet of things (IIoT), semantic technology, virtual reality, and artificial intelligence (AI), are applied in digital twin research. This signifies a future technological trend.

\section{Multi-approach based modeling}

Multiple approaches [3, 4] are adopted to model digital and virtual twins based on geometry, simulation, and data. Specifically, techniques such as generative design, 3D scan, deep learning, knowledge graph, and mesh-free simulation become popular. These techniques improve the digital and virtual model construction on precision and efficiency.

\section{IIoT-based sensor technology}

IIoT is a "system comprising networked smart objects, cyber-physical assets, associated generic information technologies and optional cloud or edge computing platforms" [8]. It supports sensors to be distributed across a wider geographic locations and different industries with an integrated fashion. It allows digital twins to collect abundant data of physical objects and give feedbacks. It enables data analytics based on data fusion of multi-channel data to enhance the ability of real-time simulation and decision making.

\section{- Semantics-based digital twin development}

Semantic technologies play an important role in digital twin research. For example, knowledge representation techniques of collaborative concepts [9], linked data and web ontologies [10] support physical, digital and virtual twins for model interoperability, heterogeneous data, and process 
integration. They allow the objects and processes to be digitally threaded and integrated from local to global and from past to present to realize interoperation across models, processes, industries, and histories [11]. They enable semantic, flexible, and real-time services in all aspects to shape a holistic future ecosystem.

\section{AI-based digital twin management}

AI technology fused by the methods of machine learning, big data, service computing, semantic techniques, IIoT, and autonomous robot enables digital twins to be flexibly constructed, real-time sensed, and deeply learnt for many purposes [12]. For example, it allows digital twins to continuously evaluate data, detect anomaly, automate business processes, answer questions, simulate situations, and support decision-making and optimization.

\section{Reality-based human-computer interaction}

A trend of interactions between human and digital/virtual twins is through augmented, virtual, and mixed reality in a 3D space [5]. These technologies provide users with immersive interaction ability in all aspects of seeing, hearing, touching, smelling, and tasting.

To sum up, we believe that future digital twins will be evolved toward a holistic virtual world, that is, a two-level virtual world will be generated by accurately mapping the digital twins onto the physical twins of real world and mapping the virtual twins onto the imaginations of the mind world of human users.

\section{References}

1. Tao F, Zhang H, Liu A, Nee AYC (2019) Digital twin in industry: state-of-the-art. IEEE Trans Ind Inf 15(4):2405-2415

2. Tao F, Qi Q (2019) Make more digital twins. Nature 573:490-491

3. Liu M, Fang S, Dong H, Xu C (2020) Review of digital twin about concepts, technologies, and industrial applications. J Manuf Syst. https://doi.org/10.1016/j.jmsy.2020.06.017

4. Liu C, Jiang P, Jiang W (2020) Web-based digital twin modeling and remote control of cyber-physical production systems. Robot Comput Integr Manuf 64:101956

5. Saddik AE (2018) Digital twins: the convergence of multimedia technologies. IEEE Multimed 25(2):87-92

6. Saracco R (2019) Digital twins: bridging physical space and cyberspace. Computer 52(12):58-64

7. Voosen P (2020) Europe builds 'digital twin' of Earth to hone climate forecasts. Science 370(6512):16-17

8. Boyes H, Hallaq B, Cunningham J, Watson T (2018) The industrial internet of things (IIoT): an analysis framework. Comput Ind 101:1-12

9. Xiao G, Guo J, Xu L, Gong Z (2014) User Interoperability with heterogeneous IoT devices through transformation. IEEE Trans Ind Inf 10(2): 1486-1496

10. Boje C, Guerriero A, Kubicki S, Rezgui Y (2020) Towards a semantic construction digital twin: directions for future research. Autom Constr 114:103179

11. Kwon S, Monnier LV, Barbau R, Bernstein WZ (2020) Enriching standards-based digital thread by fusing as-designed and as-inspected data using knowledge graphs. Adv Eng Inform 46:101102

12. He B, Bai K (2020) Digital twin-based sustainable intelligent manufacturing: a review. Adv Manuf. https://doi.org/10.1007/ s40436-020-00302-5 